PROCEEDINGS OF THE

AMERICAN MATHEMATICAL SOCIETY

Volume 132, Number 5, Pages 1419-1428

S 0002-9939(03)07275-7

Article electronically published on November 4, 2003

\title{
COMPLETELY RANK NONINCREASING LINEAR MAPS ON NEST ALGEBRAS
}

\author{
JINCHUAN HOU AND JIANLIAN CUI
}

(Communicated by David R. Larson)

\begin{abstract}
In this paper, the completely rank nonincreasing bounded linear maps on nest algebras acting on separable Hilbert spaces are characterized, and an affirmative answer to a problem posed by Hadwin and Larson is given for the case of such nest algebras.
\end{abstract}

\section{INTRODUCTION}

Let $X$ and $Y$ be Banach spaces over $\mathbb{F}(\mathbb{F}=\mathbb{R}$ or $\mathbb{C}$, the real or complex field). Let $\mathcal{B}(X, Y)(\mathcal{B}(X)$, if $X=Y)$ denote the Banach space (Banach algebra) of all bounded linear operators from $X$ into $Y$ and $\mathcal{F}(X, Y)(\mathcal{F}(X)$ if $X=Y)$ the subspace (ideal) of all finite rank operators in $\mathcal{B}(X, Y)(\mathcal{B}(X))$. For a linear subspace $\mathcal{A} \subseteq \mathcal{B}(X)$, a linear map $\Phi: \mathcal{A} \rightarrow \mathcal{B}(Y)$ is called rank nonincreasing (resp., rank preserving) if $\operatorname{rank}(\Phi(A)) \leq \operatorname{rank}(A)$ (resp., $\operatorname{rank}(\Phi(A))=\operatorname{rank}(A)$ ) for every $A$ in $\mathcal{A}$, where the rank of an operator is the dimension of its range; $\Phi$ is called completely rank nonincreasing (resp., completely rank preserving) if for every positive integer $n$, $\Phi_{n}: \mathcal{A} \otimes M_{n}(\mathbb{F}) \rightarrow \mathcal{B} \otimes M_{n}(\mathbb{F})$ is rank nonincreasing (resp., rank preserving), where $\mathcal{A} \otimes M_{n}(\mathbb{F})=\left\{\left(T_{i j}\right)_{n \times n} \mid T_{i j} \in \mathcal{A}\right\}$ with a suitable norm and $\Phi_{n}$ is defined by $\Phi_{n}\left(\left(T_{i j}\right)_{n \times n}\right)=\left(\Phi\left(T_{i j}\right)\right)_{n \times n}$.

Rank preserving linear maps and rank nonincreasing linear maps play important roles in the study of both homomorphisms and linear preservers on operator algebras. This is because in many cases the study of these maps can be reduced to the discussion of rank preserving or rank nonincreasing linear maps (e.g., see [1, [2], 4], [5, [7, 8], 9], [10] and the references therein). A characterization of rank preserving or rank nonincreasing linear maps on $\mathcal{F}(X)$ or $\mathcal{B}(X)$ was given in [7]. For nest algebra cases, some work has been devoted to rank preserving linear maps on upper triangular matrix algebras (see [1], [9]); for the infinite-dimensional situations, some results on nest algebras with some special kinds of nests may be found in [2, 10]. We gave in [8] a thorough discussion of rank preserving linear maps on general nest algebras of Banach space operators. However, it seems difficult to give an applicable characterization for rank nonincreasing linear maps on nest algebras. As far as we know, there have been no such results even for the upper triangular matrix algebra case. However, in this paper, we are able to get a characterization

Received by the editors October 8, 2001 and, in revised form, January 8, 2003.

2000 Mathematics Subject Classification. Primary 47B48, 47L35.

Key words and phrases. Nest algebras, rank, linear maps.

This work is supported by NNSFC and PNSFS. 
of linear maps on nest algebras acting on a separable Hilbert space when the maps are bounded and completely rank nonincreasing.

Our discussion is also motivated by the following problem posed in [4], and in [5] for the Hilbert space case. This problem is still open even for the case that $X$ and $Y$ are finite dimensional, and is closely related to another open problem of characterizing the asymptotic joint-similarity of operator tuples (see [5]).

Problem H. Let $\mathcal{G} \subseteq \mathcal{B}(X)$ be a linear subspace and $\Phi: \mathcal{G} \rightarrow \mathcal{B}(Y)$ be a linear map, where $X$ and $Y$ are Banach spaces over $\mathbb{F}$. Assuming that $\Phi$ is bounded and completely rank nonincreasing, must there exist nets $\left\{A_{\lambda} \mid \lambda \in \Lambda\right\} \subset \mathcal{B}(X, Y)$ and $\left\{B_{\lambda} \mid \lambda \in \Lambda\right\} \subset \mathcal{B}(Y, X)$ such that for every $T \in \mathcal{G}, \Phi(T)=\lim _{\lambda} A_{\lambda} T B_{\lambda}$ (in WOT or SOT)? Here, WOT and SOT stand for the weak operator topology and the strong operator topology, respectively.

Note that if $\Phi$ has the form stated in the problem, then it is surely completely rank nonincreasing. It was shown in 4 that the answer to the above problem is affirmative in the cases that $\mathcal{G}=\mathcal{F}(X)$ or $\mathcal{B}(X)$, or $\mathcal{G}$ is a semi-simple subalgebra of $M_{n}(\mathbb{F})$. For the case that $\mathcal{G}$ is an upper triangular operator matrix algebra, Problem $\mathrm{H}$ was answered positively in [2]. Our main result in this paper gives an affirmative answer to Problem $\mathrm{H}$ for the case that $\mathcal{G}$ is a nest algebra acting on a separable Hilbert space.

We mention here that the problem of characterizing the elementary operators on operator algebras is also concerned with the discussion of completely rank nonincreasing linear maps. Recall that a linear map $\Phi$ from an operator space $\mathcal{A} \subseteq \mathcal{B}(X)$ into $\mathcal{B}(Y)$ is called an elementary operator if there are operators $A_{1}, \ldots, A_{r} \in$ $\mathcal{B}(X, Y)$ and $B_{1}, \ldots, B_{r} \in \mathcal{B}(Y, X)$ such that $\Phi(T)=A_{1} T B_{1}+\cdots+A_{r} T B_{r}$ for all $T \in \mathcal{A}$. The integer $l(\Phi)=\min \left\{r \mid \Phi(\cdot)=\sum_{i=1}^{r} A_{i}(\cdot) B_{i}\right\}$ is called the length of $\Phi$. Note that each elementary operator $\Phi$ of length $k$ is $k$-rank nonincreasing, i.e., $\operatorname{rank}(\Phi(T)) \leq k(\operatorname{rank}(T))$ for all $T \in \mathcal{A}$. In [5], the completely rank nonincreasing linear maps and completely $k$-rank nonincreasing linear maps on $\mathcal{B}(H)$, where $H$ is a Hilbert space, were introduced and discussed, and furthermore, a characterization of elementary operators on $\mathcal{B}(H)$ was given, which states that a linear map on $\mathcal{B}(H)$ is an elementary operator of length at most $k$ if and only if it is $\sigma$-weakly continuous, completely bounded and completely $k$-rank nonincreasing. For the case of general Banach spaces, a stronger result was obtained in [4, which states that a linear map from $\mathcal{B}(X)$ into itself is an elementary operator of length at most $k$ if and only if it is $\sigma$-weakly continuous and completely $k$-rank nonincreasing, while Problem $\mathrm{H}$ asks whether every bounded and completely rank nonincreasing linear map acting on a subspace of $\mathcal{B}(X)$ is a strong limit of a net of elementary operators of length 1 .

From now on, $H$ and $K$ will denote two separable Hilbert spaces over $\mathbb{F}$ with inner product denoted by $\langle\cdot, \cdot\rangle$. A nest on $H$ is a chain $\mathcal{N}$ of closed (under norm topology) linear subspaces of $H$ containing $\{0\}$ and $H$, which is closed under the formation of arbitrary closed linear span (written by $\bigvee$ ) and intersection (written by $\Lambda$ ). $A \lg \mathcal{N}$ stands for the associated nest algebra, which is the set of all operators $T$ in $\mathcal{B}(H)$ such that $T N \subseteq N$ for every element $N \in \mathcal{N}$. Denote $\operatorname{Alg}_{\mathcal{F}} \mathcal{N}=\operatorname{Alg} \mathcal{N} \cap \mathcal{F}(H)$. For $N \in \mathcal{N}$, set $N_{-}=\bigvee\{M \in \mathcal{N} \mid M \subset N\}$ and $N_{+}=\bigwedge\{M \in \mathcal{N} \mid N \subset M\}$, where the notation " $\subset$ " stands for the proper contained relation between sets. Define $0_{-}=0$ and $H_{+}=H$. As usual, $N^{\perp}$ is the orthogonal complement of $N$ and $\mathbb{N}$ is the set of all natural numbers. For $x, f \in H$, the rank- 1 operator defined by 
$y \longmapsto\langle y, f\rangle x$ will be denoted by $x \otimes f$ and $x \otimes f \in \operatorname{Alg}_{\mathcal{F}} \mathcal{N}$ if and only if there exists $N \in \mathcal{N}$ such that $x \in N$ and $f \in N_{-}^{\perp}$ (ref. [3]).

\section{MAIN RESUlT AND ITS PROOF}

In this section we state the main result and give its proof.

Main Theorem. Let $\Phi: \operatorname{Alg} \mathcal{N} \rightarrow \mathcal{B}(K)$ be a bounded linear map. Then the following are equivalent:

(1) $\Phi$ is completely rank nonincreasing.

(2) There exist bounded operator nets $\left\{A_{\lambda}\right\} \subset \mathcal{B}(H, K)$ and $\left\{B_{\lambda}\right\} \subset \mathcal{B}(K, H)$ such that $\Phi(T)=s$ - $\lim _{\lambda} A_{\lambda} T B_{\lambda}$ for every $T \in \operatorname{Alg} \mathcal{N}$, where $s$-lim denotes the limit under SOT. Moreover, if $\Phi$ is unital, then $\left\{B_{\lambda}\right\}$ can been chosen so that $B_{\lambda}=A_{\lambda}^{-1}$.

Proof. Only $(1) \Rightarrow(2)$ needs to be checked, and the proof will be divided into several steps. Assume (1) holds. Then $\Phi_{2}$ is rank-1 nonincreasing.

For $x \in H$, let $L_{x}^{\mathcal{N}}=\left\{x \otimes f \in \operatorname{Alg}_{\mathcal{F}} \mathcal{N} \mid f \in H\right\}$ and $L_{x}=\{x \otimes f \mid f \in H\}$.

Step 1. We first show that, for any $x \in H$, there is $y(x) \in K$ such that $\Phi\left(L_{x}^{\mathcal{N}}\right) \subseteq L_{y(x)}$ and hence $\Phi(x \otimes f)=y(x) \otimes g_{x}(f)$ for every $x \otimes f \in \operatorname{Alg}_{\mathcal{F}} \mathcal{N}$.

If $\Phi\left(L_{x}^{\mathcal{N}}\right)=0$, we always put $y(x)=0$; if $\Phi\left(L_{x}^{\mathcal{N}}\right) \neq 0$, then there exists $f_{0} \in H$ such that $\Phi\left(x \otimes f_{0}\right)=y_{0} \otimes g_{0} \neq 0$. For any $f \in H$ such that $x \otimes f \in \operatorname{Alg}_{\mathcal{F}} \mathcal{N}$, let $\Phi(x \otimes$ $f)=y \otimes g$. Then $\left(\begin{array}{cc}x \otimes f_{0} & x \otimes f \\ 0 & 0\end{array}\right)$ is of rank one and hence $\left(\begin{array}{cc}y_{0} \otimes g_{0} & y \otimes g \\ 0 & 0\end{array}\right)$ is of rank one. This implies that $y \in \operatorname{span}\left\{y_{0}\right\}$. So $\Phi(x \otimes f) \in L_{y_{0}}$. Taking $y(x)=y_{0}$, we have $\Phi\left(L_{x}^{\mathcal{N}}\right) \subseteq L_{y(x)}$.

Since $H$ is separable, there exists a closed subset $\Gamma$ of the interval $[0,1]$ with $0,1 \in \Gamma$ such that $\Gamma$ has the same order type as $\mathcal{N}$. In the sequel, we index $\mathcal{N}$ by $\Gamma$, i.e., write $\mathcal{N}=\left\{N_{\gamma} \mid \gamma \in \Gamma\right\}$.

Step 2. Let $\beta_{10}=0, \Lambda_{11}=\left\{\gamma \geq \beta_{10} \mid \Phi\left(L_{x}^{\mathcal{N}}\right)=0\right.$ whenever $\left.x \in N_{\gamma}\right\}$ and $\alpha_{11}=\sup \left\{\gamma \mid \gamma \in \Lambda_{11}\right\}$. Then $\alpha_{11} \in \Lambda_{11}$.

If $\alpha_{11} \notin \Lambda_{11}$, then there exists $x_{0} \in N_{\alpha_{11}}$ and $f_{0} \in H$ such that $x_{0} \otimes f_{0} \in L_{x_{0}}^{\mathcal{N}}$ and $\Phi\left(x_{0} \otimes f_{0}\right) \neq 0$. Take an increasing sequence $\left\{\gamma_{n}\right\} \subset \Lambda_{11}$ such that $\lim _{n \rightarrow \infty} \gamma_{n}=\alpha_{11}$ and denote $L_{n}=N_{\gamma_{n}} \ominus N_{\gamma_{n-1}}\left(N_{\gamma_{0}}=0\right)$. Then $N_{\alpha_{11}}=\bigoplus_{n=1}^{\infty} L_{n}$ and $x_{0}=\bigoplus_{n=1}^{\infty} \xi_{n}$, where $\xi_{n} \in L_{n} \subset N_{\gamma_{n}}$. Since $x_{0} \otimes f_{0} \in \operatorname{Alg}_{\mathcal{F}} \mathcal{N}$, we have $f_{0} \in\left(N_{\alpha_{11}}\right)_{-}^{\perp}$. Thus for every $n, \xi_{n} \otimes f_{0} \in \operatorname{Alg}_{\mathcal{F}} \mathcal{N}$ and $\Phi\left(\xi_{n} \otimes f_{0}\right)=0$. The boundedness of $\Phi$ leads to $\Phi\left(x_{0} \otimes f_{0}\right)=\lim _{n \rightarrow \infty} \Phi\left(\sum_{k=1}^{n} \xi_{n} \otimes f_{0}\right)=0$, a contradiction.

Step 3. Let $\Delta_{11}=\left\{\gamma>\alpha_{11} \mid \Phi(x \otimes f)=0\right.$ whenever $x \in N_{\gamma} \ominus N_{\alpha_{11}}$ and $\left.f \in N_{\gamma}^{\perp}\right\}$ and $\beta_{11}=\inf \left\{\gamma \mid \gamma \in \Delta_{11}\right\}$. Then $\beta_{11} \in \Delta_{11}$.

On the contrary, if $\beta_{11} \notin \Delta_{11}$, then there exist $x_{0} \in N_{\beta_{11}} \ominus N_{\alpha_{11}}$ and $f_{0} \in N_{\beta_{11}}^{\perp}$ such that $\Phi\left(x_{0} \otimes f_{0}\right) \neq 0$. Thus we may take a decreasing sequence $\left\{\gamma_{n}\right\} \subset \Delta_{11}$ such that $\lim _{n \rightarrow \infty} \gamma_{n}=\beta_{11}$. Then, a similar argument just as in step 2 will lead to a contradiction.

Step 4. There exist operator sequences $\left\{A_{11}^{(n)}\right\}_{n=1}^{\infty},\left\{C_{11}^{(n)}\right\}_{n=1}^{\infty} \subset \mathcal{B}\left(N_{\beta_{11}} \odot\right.$ $\left.N_{\beta_{10}}, K\right)$ such that $\Phi(x \otimes f)=\lim _{n \rightarrow \infty} A_{11}^{(n)} x \otimes C_{11}^{(n)} f$ holds for all $x \otimes f \in \operatorname{Alg}_{\mathcal{F}} \mathcal{N}$ with $x, f \in N_{\beta_{11}} \ominus N_{\beta_{10}}$, and $\Phi(x \otimes f)=0$ for every $x \in N_{\beta_{11}} \odot N_{\beta_{10}}$ and $f \in\left(N_{\beta_{11}}\right)^{\perp}$.

To prove this assertion, we have to consider, respectively, the following four cases:

Case $1^{\circ} .\left(N_{\beta_{11}}\right)_{-}=N_{\beta_{11}}$ and $\left(N_{\alpha_{11}}\right)_{+}=N_{\alpha_{11}}$;

Case $2^{\circ} .\left(N_{\beta_{11}}\right)_{-} \subset N_{\beta_{11}}$ and $\left(N_{\alpha_{11}}\right)_{+}=N_{\alpha_{11}}$; 
Case $3^{\circ} \cdot\left(N_{\beta_{11}}\right)_{-}=N_{\beta_{11}}$ and $\left(N_{\alpha_{11}}\right)_{+} \supset N_{\alpha_{11}}$

Case $4^{\circ} .\left(N_{\beta_{11}}\right)_{-} \subset N_{\beta_{11}}$ and $\left(N_{\alpha_{11}}\right)_{+} \supset N_{\alpha_{11}}$.

We only check the assertion in step 4 for case $1^{\circ}$ here. The other cases can be treated similarly. Assume that case $1^{\circ}$ occurs. We first claim that there exists an increasing sequence $\left\{\delta_{n}\right\} \subset \Gamma$ converging to $\beta_{11}$, a decreasing sequence $\left\{\gamma_{n}\right\} \subset$ $\Gamma$ converging to $\alpha_{11}$ and vectors $x_{n} \in N_{\gamma_{n}} \ominus N_{\alpha_{11}}, f_{n} \in N_{\beta_{11}} \ominus N_{\delta_{n}}$ such that $\Phi\left(x_{n} \otimes f_{n}\right)=y_{n} \otimes g_{n}\left(f_{n}\right) \neq 0$ for every $n \in \mathbb{N}$ (we may require that $\gamma_{n}<\delta_{m}$ for any $n, m \in \mathbb{N}$ ). If the claim is not true, then there exist $\gamma_{0}, \delta_{0}$ in $\Gamma$ satisfying $\alpha_{11}<\gamma_{0}<\delta_{0}<\beta_{11}$ such that $\Phi(x \otimes f)=0$ whenever $x \in N_{\gamma_{0}} \ominus N_{\alpha_{11}}$ and $f \in N_{\beta_{11}} \ominus N_{\delta_{0}}$. Let $\Delta_{11}^{\prime}=\left\{\gamma \geq \gamma_{0} \mid \Phi(x \otimes f)=0\right.$ whenever $x \in N_{\gamma_{0}} \ominus N_{\alpha_{11}}$ and $\left.f \in N_{\gamma}^{\perp}\right\}$ and let $\beta_{11}^{\prime}=\inf \left\{\gamma \mid \gamma \in \Delta_{11}^{\prime}\right\}$. It is clear that $\gamma_{0} \leq \beta_{11}^{\prime} \leq \delta_{0}<\beta_{11}$. Now let us show that $\beta_{11}^{\prime} \in \Delta_{11}$. Assume, on the contrary, that $\beta_{11}^{\prime} \notin \Delta_{11}$. Then there exist $x_{0} \in N_{\beta_{11}^{\prime}} \ominus N_{\alpha_{11}}$ and $f_{0} \in N_{\beta_{11}} \ominus N_{\beta_{11}^{\prime}}$ such that $\Phi\left(x_{0} \otimes f_{0}\right) \neq 0$. We will, respectively, deduce a contradiction in both cases $\left(N_{\beta_{11}^{\prime}}\right)_{-}=N_{\beta_{11}^{\prime}}$ and $\left(N_{\beta_{11}^{\prime}}\right)_{-} \neq N_{\beta_{11}^{\prime}}$. If $\left(N_{\beta_{11}^{\prime}}\right)_{-}=N_{\beta_{11}^{\prime}}$, we may require $x_{0} \in N_{\gamma} \ominus N_{\alpha_{11}}$ for some $\gamma$ with $\alpha_{11}<\gamma<\beta_{11}^{\prime}$. In fact, there exists an increasing sequence $\left\{\theta_{n}\right\} \subset \Gamma$ converging to $\beta_{11}^{\prime}$. Let $L_{n}=N_{\theta_{n}} \ominus N_{\theta_{n-1}}\left(N_{\theta_{0}}=0\right)$. Then $N_{\beta_{11}^{\prime}}=\bigoplus_{n=1}^{\infty} L_{n}$ and $x_{0}=\bigoplus_{n=1}^{\infty} \xi_{n}=\lim _{l \rightarrow \infty} \bigoplus_{n=1}^{l} \xi_{n}$, where $\xi_{n} \in L_{n} \subset N_{\theta_{n}}$. Since $\Phi$ is bounded and $\Phi\left(x_{0} \otimes f_{0}\right) \neq 0$, there must be a $k \in \mathbb{N}$ such that $\Phi\left(\sum_{n=1}^{k} \xi_{n} \otimes f_{0}\right) \neq 0$. It is clear that $x_{k}=\sum_{n=1}^{k} \xi_{n} \in N_{\theta_{k}} \subset N_{\beta_{11}^{\prime}}$. So we may take $\gamma=\theta_{k}<\beta_{11}^{\prime}$. By the definition of $\beta_{11}^{\prime}$, for $\gamma<\beta_{11}^{\prime}$, there exist $x_{1} \in N_{\gamma_{0}} \ominus N_{\alpha_{11}}$ and $f_{1} \in N_{\gamma}^{\perp}$ such that $\Phi\left(x_{1} \otimes f_{1}\right) \neq 0$. Note that $x_{0} \otimes f_{1}, x_{1} \otimes f_{0} \in \operatorname{Alg}_{\mathcal{F}} \mathcal{N}$. So

$$
F=\left(\begin{array}{cc}
x_{0} \otimes f_{0} & x_{0} \otimes f_{1} \\
x_{1} \otimes f_{0} & x_{1} \otimes f_{1}
\end{array}\right) \in \operatorname{Alg}_{\mathcal{F}} \mathcal{N} \otimes M_{2}(\mathbb{F})
$$

is of rank one. However, $\Phi\left(x_{1} \otimes f_{0}\right)=0$ implies that $\operatorname{rank}\left(\Phi_{2}(F)\right)=2$, a contradiction. If $\left(N_{\beta_{11}^{\prime}}\right)_{-} \neq N_{\beta_{11}^{\prime}}$, then we can take either $x_{0} \in\left(N_{\beta_{11}^{\prime}}\right)_{-} \ominus N_{\alpha_{11}}$ or $x_{0} \in N_{\beta_{11}^{\prime}} \ominus\left(N_{\beta_{11}^{\prime}}\right)_{-}$. For the former, taking $N_{\gamma}=\left(N_{\beta_{11}^{\prime}}\right)_{-}$, a similar argument as above leads to a contradiction. For the latter, the definition of $\beta_{11}^{\prime}$ implies that there exist $x_{1} \in N_{\gamma_{0}} \ominus N_{\alpha_{11}}$ and $f_{1} \in N_{\beta_{11}^{\prime}} \ominus\left(N_{\beta_{11}^{\prime}}\right)_{-}$such that $\Phi\left(x_{1} \otimes f_{1}\right) \neq 0$. Since $f_{1} \in\left(N_{\beta_{11}^{\prime}}\right)_{-}^{\perp}$ and $\gamma_{0}<\beta_{11}^{\prime}$, we still have $x_{0} \otimes f_{1}, x_{1} \otimes f_{0} \in \operatorname{Alg} \mathcal{F} \mathcal{N}$. From the facts that $\Phi\left(x_{1} \otimes f_{0}\right)=0$ and $\Phi_{2}$ is rank-1 nonincreasing, we get a contradiction again by considering the operator matrix $\left(\begin{array}{cc}x_{0} \otimes f_{0} & x_{0} \otimes f_{1} \\ x_{1} \otimes f_{0} & x_{1} \otimes f_{1}\end{array}\right)$. So $\beta_{11}^{\prime} \in \Delta_{11}$. However, this implies $\beta_{11} \leq \beta_{11}^{\prime}<\beta_{11}$, which is impossible. Hence the claim is true.

Now assume that $\left\{\delta_{n}\right\},\left\{\gamma_{n}\right\},\left\{x_{n}\right\}$ and $\left\{f_{n}\right\}$ just as in the preceding paragraph have been taken. For every $x_{n} \otimes f \in L_{x_{n}}^{\mathcal{N}}$, by step $1, \Phi\left(x_{n} \otimes f\right)=y_{n} \otimes g_{n}(f)$. It is clear that $g_{n}$ is linear on $D\left(x_{n}\right)=\left\{f \mid x_{n} \otimes f \in L_{x_{n}}^{\mathcal{N}}\right\}$ and $\left(N_{\gamma_{n}}\right)_{-}^{\perp} \subset D\left(x_{n}\right)$. Also note that, by the boundedness of $\Phi$ and the closed graph theorem, $\left.g_{n}\right|_{\left(N_{\gamma_{n}}\right)_{-}}$: $\left(N_{\gamma_{n}}\right)_{-}^{\perp} \rightarrow K$ is a bounded linear map.

Next we claim that, for any $k \in \mathbb{N}$, $\operatorname{dim} \bigvee\left\{\left.g_{n}\right|_{\left(N_{\gamma_{k}}\right)_{-}^{\perp}}\right\}_{n=k}^{\infty}=1$. Otherwise, there exist $n_{i}, n_{j} \geq k$ such that $\left.g_{n_{i}}\right|_{\left(N_{\gamma_{k}}\right)_{\perp}}$ and $\left.g_{n_{j}}\right|_{\left(N_{\gamma_{k}}\right)_{\perp}^{\perp}}$ are linearly independent. Say $n_{j}>n_{i}$, thus $\delta_{n_{i}}<\delta_{n_{j}}$ and $\gamma_{n_{i}}>\gamma_{n_{j}}$, which imply that $x_{n_{i}} \otimes f_{n_{j}}, x_{n_{j}} \otimes f_{n_{i}} \in$ 
$\operatorname{Alg}_{\mathcal{F}} \mathcal{N}$. Since

$$
\left(\begin{array}{cc}
x_{n_{i}} \otimes f_{n_{i}} & x_{n_{i}} \otimes f_{n_{j}} \\
x_{n_{j}} \otimes f_{n_{i}} & x_{n_{j}} \otimes f_{n_{j}}
\end{array}\right)
$$

is of rank one,

$$
\left(\begin{array}{ll}
y_{n_{i}} \otimes g_{n_{i}}\left(f_{n_{i}}\right) & y_{n_{i}} \otimes g_{n_{i}}\left(f_{n_{j}}\right) \\
y_{n_{j}} \otimes g_{n_{j}}\left(f_{n_{i}}\right) & y_{n_{j}} \otimes g_{n_{j}}\left(f_{n_{j}}\right)
\end{array}\right)
$$

is of rank one, too. So there must be $g_{n_{i}}\left(f_{n_{i}}\right)=g_{n_{j}}\left(f_{n_{i}}\right)$ and $g_{n_{i}}\left(f_{n_{j}}\right)=g_{n_{j}}\left(f_{n_{j}}\right)$. For any $f \in\left(N_{\gamma_{k}}\right)_{-}^{\perp}$, we have $x_{n_{i}} \otimes f, x_{n_{j}} \otimes f \in \operatorname{Alg}_{\mathcal{F}} \mathcal{N}$. Again, the rank-oneness of

$$
\left(\begin{array}{ll}
x_{n_{i}} \otimes f & x_{n_{i}} \otimes f_{n_{j}} \\
x_{n_{j}} \otimes f & x_{n_{j}} \otimes f_{n_{j}}
\end{array}\right)
$$

implies that

$$
\left(\begin{array}{cc}
y_{n_{i}} \otimes g_{n_{i}}(f) & y_{n_{i}} \otimes g_{n_{i}}\left(f_{n_{j}}\right) \\
y_{n_{j}} \otimes g_{n_{j}}(f) & y_{n_{j}} \otimes g_{n_{j}}\left(f_{n_{j}}\right)
\end{array}\right)
$$

is of rank one, too. It follows that $g_{n_{i}}(f)=g_{n_{j}}(f)$ for all $f \in\left(N_{\gamma_{k}}\right)_{-}^{\perp}$ and therefore $\left.g_{n_{i}}\right|_{\left(N_{\gamma_{k}}\right)_{\perp}}=\left.g_{n_{j}}\right|_{\left(N_{\gamma_{k}}\right)_{\perp}}$, a contradiction. Hence for any natural number $n \geq k$, $\left.g_{n}\right|_{\left(N_{\gamma_{k}}\right) \perp}=\left.g_{k}\right|_{\left(N_{\gamma_{k}}\right) \perp}$, that is, the claim holds.

Similarly, for every $n \in \mathbb{N}$, one gets a linear transformation $y_{n}: D\left(f_{n}\right)=\{x \mid$ $\left.x \otimes f_{n} \in \operatorname{Alg}_{\mathcal{F}} \mathcal{N}\right\} \rightarrow K$ such that $\Phi\left(x \otimes f_{n}\right)=y_{n}(x) \otimes g_{n}\left(f_{n}\right),\left.y_{n}\right|_{N_{\delta_{n}}}$ is bounded and $\left.y_{m}\right|_{N_{\delta_{n}}}=\left.y_{n}\right|_{N_{\delta_{n}}}$ if $m>n$.

Now, define $w: \bigcup_{n=1}^{\infty}\left\{N_{\delta_{n}}\right\} \rightarrow K$ by $\left.w\right|_{N_{\delta_{n}}}=\left.y_{n}\right|_{N_{\delta_{n}}}$ and $g: \bigcup_{n=1}^{\infty}\left\{\left(N_{\gamma_{n}}\right)_{-}^{\perp}\right\} \rightarrow$ $K$ by $\left.g\right|_{\left(N_{\gamma_{n}}\right)_{\perp}}=\left.g_{n}\right|_{\left(N_{\gamma_{n}}\right)_{\perp}}$. Then $w$ and $g$ are linear. For any $x, f \in N_{\beta_{11}} \ominus N_{\alpha_{11}}$ so that $x \otimes f \in \operatorname{Alg}_{\mathcal{F}} \mathcal{N}$, there exists $\gamma \in \Gamma$ such that $x \in N_{\gamma} \ominus N_{\alpha_{11}}$ and $f \in$ $N_{\beta_{11}} \ominus\left(N_{\gamma}\right)_{-}$. We may require that $\gamma=\min \left\{\delta \mid x \in N_{\delta} \ominus N_{\alpha_{11}}\right\}$. Since the sequence $\left\{\delta_{n}\right\}$ increasingly converges to $\beta_{11}$ and $\left\{\gamma_{n}\right\}$ decreasingly converges to $\alpha_{11}$, there exists $n \in \mathbb{N}$ satisfying $\gamma_{n}<\gamma<\delta_{n}$. Obviously $x_{n} \otimes f, x \otimes f_{n} \in \operatorname{Alg}_{\mathcal{F}} \mathcal{N}$. Hence

$$
\left(\begin{array}{cc}
x_{n} \otimes f_{n} & x_{n} \otimes f \\
x \otimes f_{n} & x \otimes f
\end{array}\right)
$$

is of rank one and, consequently,

$$
\left(\begin{array}{cc}
y_{n} \otimes g_{n}\left(f_{n}\right) & y_{n} \otimes g_{n}(f) \\
y_{n}(x) \otimes g_{n}\left(f_{n}\right) & y(x) \otimes g_{x}(f)
\end{array}\right)
$$

is of rank one. So $y(x)$ and $y_{n}(x)$ are linearly dependent. Assume that both $y(x)$ and $y_{n}(x)$ are nonzero. Then we may suppose that $y(x)=y_{n}(x)=w(x)$ and $g_{x}(f)=g_{n}(f)=g(f)$. If $y(x)=0$, we must have $y_{n}(x)=0$ since $g_{n}\left(f_{n}\right) \neq 0$, and we may still take $g_{x}(f)=g_{n}(f)$; if $y_{n}(x)=0$ but $y(x) \neq 0$, then there must be $g_{x}(f) \equiv 0$ for all $f \in\left(N_{\gamma}\right)_{-}^{\perp}$, which implies that $\Phi\left(L_{x}^{\mathcal{N}}\right)=0$, and hence $y(x)=0$ by step 1 , which contradicts the assumption $y(x) \neq 0$. Therefore, $\Phi(x \otimes f)=$ $y_{n}(x) \otimes g_{n}(f)=w(x) \otimes g(f)$ for any $x, f \in N_{\beta_{11}} \ominus N_{\alpha_{11}}$ with $x \otimes f \in \operatorname{Alg}_{\mathcal{F}} \mathcal{N}$.

For each $n$, define respectively $A_{11}^{(n)}$ and $C_{11}^{(n)}$ from $\left(N_{\beta_{11}} \ominus N_{\beta_{10}}\right)$ into $K$ by

$$
\begin{gathered}
A_{11}^{(n)} x= \begin{cases}w(x) & \text { if } x \in N_{\delta_{n}} \ominus N_{\beta_{10}}, \\
0 & \text { if } x \in N_{\beta_{11}} \ominus N_{\delta_{n}} ;\end{cases} \\
C_{11}^{(n)} f= \begin{cases}g(f) & \text { if } f \in N_{\beta_{11}} \ominus\left(N_{\gamma_{n}}\right)_{-}, \\
0 & \text { if } f \in N_{\gamma_{n}} \ominus N_{\beta_{10} .} .\end{cases}
\end{gathered}
$$


Clearly, $A_{11}^{(n)}, C_{11}^{(n)} \in \mathcal{B}\left(N_{\beta_{11}} \ominus N_{\beta_{10}}, K\right)$ for any $n \in \mathbb{N}$ and $\Phi(x \otimes f)=\lim _{n \rightarrow \infty} A_{11}^{(n)} x \otimes$ $C_{11}^{(n)} f$ for every $x \otimes f \in \operatorname{Alg} \mathcal{F} \mathcal{N}$ with $x, f \in N_{\beta_{11}} \odot N_{\beta_{10}}$, finishing the proof of step 4.

Step 5. If $\beta_{11}=1$, we stop. If not, let $\Lambda_{12}=\left\{\gamma>\beta_{11} \mid \Phi\left(L_{x}^{\mathcal{N}}\right)=0\right.$ whenever $\left.x \in N_{\gamma} \ominus N_{\beta_{11}}\right\}$. If $\Lambda_{12}=\emptyset$, let $\alpha_{12}=\beta_{11}$; if $\Lambda_{12} \neq \emptyset$, let $\alpha_{12}=\sup \left\{\gamma \mid \gamma \in \Lambda_{12}\right\}$. Similar to step 2, we have $\alpha_{12} \in \Lambda_{12}$. Hence for any $x \in N_{\alpha_{12}} \ominus N_{\beta_{11}}, \Phi\left(L_{x}^{\mathcal{N}}\right)=0$. If $\alpha_{12}=1$, we stop. If not, let $\Delta_{12}=\left\{\gamma>\alpha_{12} \mid \Phi(x \otimes f)=0\right.$ whenever $x \in N_{\gamma} \ominus N_{\alpha_{12}}$ and $\left.f \in N_{\gamma}^{\perp}\right\}$ and $\beta_{12}=\inf \left\{\gamma \mid \gamma \in \Delta_{12}\right\}$; then $\beta_{12} \in \Delta_{12}$. Similar to step 4, one can show that there exist bounded operator sequences $\left\{A_{12}^{(n)}\right\},\left\{C_{12}^{(n)}\right\} \subset \mathcal{B}\left(N_{\beta_{12}} \ominus N_{\beta_{11}}\right.$, $K)$ such that $\Phi(x \otimes f)=\lim _{n \rightarrow \infty} A_{12}^{(n)} x \otimes C_{12}^{(n)} f$ for every $x \otimes f \in \operatorname{Alg}_{\mathcal{F}} \mathcal{N}$ with $x$, $f \in N_{\beta_{12}} \ominus N_{\beta_{11}}$, and $\Phi(x \otimes f)=0$ for any $x \in N_{\beta_{12}} \ominus N_{\beta_{11}}$ and $f \in\left(N_{\beta_{12}}\right)^{\perp}$.

Continuing in this way whenever it is possible, we get sequences

$$
0=\beta_{10} \leq \alpha_{11}<\beta_{11} \leq \alpha_{12}<\beta_{12} \leq \cdots \leq \alpha_{1 k}<\beta_{1 k} \leq \alpha_{1(k+1)}<\cdots
$$

and $\left\{A_{1 k}^{(n)}\right\}_{n=1}^{\infty},\left\{C_{1 k}^{(n)}\right\}_{n=1}^{\infty} \subset \mathcal{B}\left(N_{\beta_{1 k}} \ominus N_{\beta_{1(k-1)}}, K\right)$ such that for each $(1, k)$,

$$
\Phi(x \otimes f)=\lim _{n \rightarrow \infty} A_{1 k}^{(n)} x \otimes C_{1 k}^{(n)} f
$$

for every $x \otimes f \in \operatorname{Alg}_{\mathcal{F}} \mathcal{N}$ with $x, f \in N_{\beta_{1 k}} \ominus N_{\beta_{1(k-1)}}$, and $\Phi(x \otimes f)=0$ for any $x \in N_{\beta_{1 k}} \ominus N_{\beta_{1(k-1)}}$ and $f \in\left(N_{\beta_{1 k}}\right)^{\perp}$.

Step 6. If $\beta_{1 m}=1$ for some $m$, we stop; if $\beta_{1 m}<1$ but $\alpha_{1(m+1)}=1$, we stop, and let $A_{1(m+1)}^{(n)}=C_{1(m+1)}^{(n)}=0$ on $N_{\alpha_{1(m+1)}} \ominus N_{\beta_{1 m}}$. If not, we get two infinite sequences $\left\{\alpha_{1 k}\right\}_{k=1}^{\infty}$ and $\left\{\beta_{1 k}\right\}_{k=0}^{\infty}$ in $\Gamma$. Let $\beta_{20}=\sup _{k}\left\{\alpha_{1 k}\right\}$. If $\beta_{20}=1$, we stop; if $\beta_{20}<1$, in the same way just as for steps $1-5$, we get again sequences

$$
\beta_{20} \leq \alpha_{21}<\beta_{21} \leq \alpha_{22}<\beta_{22} \leq \cdots \leq \alpha_{2 k}<\beta_{2 k} \leq \alpha_{2(k+1)}<\cdots
$$

and $\left\{A_{2 k}^{(n)}\right\}_{n=1}^{\infty},\left\{C_{2 k}^{(n)}\right\}_{n=1}^{\infty} \subset B\left(N_{\beta_{2 k}} \ominus N_{\beta_{2(k-1)}}, K\right)$ such that for each $(2, k)$,

$$
\Phi(x \otimes f)=\lim _{n \rightarrow \infty} A_{2 k}^{(n)} x \otimes C_{2 k}^{(n)} f
$$

for every $x \otimes f \in \operatorname{Alg}_{\mathcal{F}} \mathcal{N}$ with $x, f \in N_{\beta_{2 k}} \ominus N_{\beta_{2(k-1)}}$, and $\Phi(x \otimes f)=0$ for any $x \in N_{\beta_{2 k}} \ominus N_{\beta_{2(k-1)}}$ and $f \in\left(N_{\beta_{2 k}}\right)^{\perp}$.

Continue the above process. Since $H$ is separable, at last we get sequences $\left\{\alpha_{i k}\right\}_{k=1}^{n_{i}}$ and $\left\{\beta_{i k}\right\}_{k=0}^{n_{i}}$ in $\Gamma\left(n_{i} \in \mathbb{N} \cup\{\infty\}\right.$ and $i=1, \ldots, \sigma$, where $\sigma$ is a countable ordinal number) such that

(1) $\beta_{i 0} \leq \alpha_{i 1}<\beta_{i 1} \leq \alpha_{i 2}<\cdots \leq \alpha_{i k}<\beta_{i k} \leq \alpha_{i(k+1)}<\cdots$;

(2) $\beta_{(i+1) 0}=\sup _{k}\left\{\alpha_{i k}\right\}, i=1,2, \ldots, \sigma$;

(3) $\sup _{i, k}\left\{\alpha_{i k}\right\}=1$; and operator sequences $\left\{A_{i k}^{(n)}\right\}_{n=1}^{\infty},\left\{C_{i k}^{(n)}\right\}_{n=1}^{\infty} \subset \mathcal{B}\left(N_{\beta_{i k}} \ominus\right.$ $\left.N_{\beta_{i(k-1)}}, K\right)$ such that for each index $(i, k)$,

$$
\Phi(x \otimes f)=\lim _{n \rightarrow \infty} A_{i k}^{(n)} x \otimes C_{i k}^{(n)} f
$$

for every $x \otimes f \in \operatorname{Alg}_{\mathcal{F}} \mathcal{N}$ with $x, f \in N_{\beta_{i k}} \ominus N_{\beta_{i(k-1)}}$, and $\Phi(x \otimes f)=0$ for any $x \in N_{\beta_{i k}} \ominus N_{\beta_{i(k-1)}}$ and $f \in\left(N_{\beta_{i k}}\right)^{\perp}$.

Let $\Omega=\left\{(i, k) \mid i=1,2, \ldots, \sigma ; k=0,1, \ldots, n_{i}\right\}$. For $(i, k)$ and $(j, l) \in \Omega$, we say $(i, k)<(j, l)$ if $i<j$ or if $i=j$ but $k<l$. Let $\Lambda=\{\lambda \mid \lambda$ is a finite subset of $\Omega\}$. Define the order " $>$ " by $\lambda_{1}>\lambda$ if and only if $\lambda_{1} \supset \lambda$. Then $\Lambda$ is a direct set. 
For $(i, k) \in \Omega$, let $H_{i k}=N_{\beta_{i k}} \ominus N_{\beta_{i(k-1)}}$. Then $H=\bigoplus_{(i, k) \in \Omega} H_{i k}$. For any $x \otimes f \in \operatorname{Alg}_{\mathcal{F}} \mathcal{N}$, let $\gamma_{x}=\inf \left\{\gamma \mid x \in N_{\gamma}\right\}$. Then $x \in N_{\gamma_{x}}$ and $f \in\left(N_{\gamma_{x}}\right)_{-}^{\perp}$. Since $\gamma_{x} \in \Gamma$, there exists $\left(i_{0}, k_{0}\right) \in \Omega$ such that $\beta_{i_{0}\left(k_{0}-1\right)}<\gamma_{x} \leq \beta_{i_{0} k_{0}}$. Hence

$$
x \in \bigoplus_{(i, k) \leq\left(i_{0}, k_{0}\right)} H_{i k} \quad \text { and } \quad f \in \bigoplus_{\left(i_{0}, k_{0}\right) \leq(i, k)} H_{i k} .
$$

Step 7. As the last step, we claim that there exist bounded operator nets $\left\{A_{\lambda} \mid \lambda \in \Lambda\right\} \subset \mathcal{B}(H, K)$ and $\left\{B_{\lambda} \mid \lambda \in \Lambda\right\} \subset \mathcal{B}(K, H)$ such that

$$
\Phi(F)=\lim _{\lambda} A_{\lambda} F B_{\lambda} \quad \text { for all } F \in \operatorname{Alg}_{\mathcal{F}} \mathcal{N} .
$$

For $\lambda \in \Lambda$, let $n_{\lambda}=\# \lambda$ (the number of members in $\lambda$ ), $t_{\lambda}=\max _{(i, k) \in \lambda}\left\{\left\|A_{i k}^{\left(n_{\lambda}\right)}\right\|\right.$, $\left.\left\|C_{i k}^{\left(n_{\lambda}\right)}\right\|\right\}$. Define $A_{\lambda}$ and $C_{\lambda}$, respectively, as follows:

$$
\begin{gathered}
A_{\lambda} x= \begin{cases}\exp \left(\alpha_{i k} n_{\lambda} t_{\lambda}\right) A_{i k}^{\left(n_{\lambda}\right)} x, & \text { if } x \in H_{i k},(i, k) \in \lambda, \\
0, & \text { if } x \in\left(\bigoplus_{(i, k) \in \lambda} H_{i k}\right)^{\perp} ;\end{cases} \\
C_{\lambda} f= \begin{cases}\exp \left(-\alpha_{i k} n_{\lambda} t_{\lambda}\right) C_{i k}^{\left(n_{\lambda}\right)} f, & \text { if } f \in H_{i k},(i, k) \in \lambda, \\
0, & \text { if } f \in\left(\bigoplus_{(i, k) \in \lambda} H_{i k}\right)^{\perp} .\end{cases}
\end{gathered}
$$

Then $A_{\lambda}, C_{\lambda} \in \mathcal{B}(H, K)$. We will show that

$$
\Phi(x \otimes f)=\lim _{\lambda} A_{\lambda} x \otimes C_{\lambda} f \quad \text { for all } x \otimes f \in \operatorname{Alg}_{\mathcal{F}} \mathcal{N}
$$

by considering four cases. To do this, fix $x \otimes f \in \operatorname{Alg} \mathcal{N}$.

Case $1^{\circ}$. There exists $\left(i_{0}, k_{0}\right) \in \Omega$ such that $x, f \in H_{i_{0} k_{0}}$. In this case we have $\Phi(x \otimes f)=\lim _{n \rightarrow \infty} A_{i_{0} k_{0}}^{(n)} x \otimes C_{i_{0} k_{0}}^{(n)} f$. For any $\varepsilon>0$, take $n_{0} \in \mathbb{N}$ such that

$$
\left\|A_{i_{0} k_{0}}^{(n)} x \otimes C_{i_{0} k_{0}}^{(n)} f-\Phi(x \otimes f)\right\|<\varepsilon
$$

whenever $n>n_{0}$. Pick $\lambda_{0} \in \Lambda$ such that $\left(i_{0}, k_{0}\right) \in \lambda_{0}$ and $n_{\lambda_{0}}=n_{0}$. Then for any $\lambda>\lambda_{0}$, we have $n_{\lambda}>n_{\lambda_{0}}=n_{0}$ and

$$
\begin{aligned}
& \left\|A_{\lambda} x \otimes C_{\lambda} f-\Phi(x \otimes f)\right\| \\
= & \left\|\exp \left(\alpha_{i_{0} k_{0}} n_{\lambda} t_{\lambda}\right) A_{i_{0} k_{0}}^{\left(n_{\lambda}\right)} x \otimes \exp \left(-\alpha_{i_{0} k_{0}} n_{\lambda} t_{\lambda}\right) C_{i_{0} k_{0}}^{\left(n_{\lambda}\right)} f-\Phi(x \otimes f)\right\| \\
= & \left\|A_{i_{0} k_{0}}^{\left(n_{\lambda}\right)} x \otimes C_{i_{0} k_{0}}^{\left(n_{\lambda}\right)} f-\Phi(x \otimes f)\right\|<\varepsilon .
\end{aligned}
$$

Case $2^{\circ} . x \in H_{i k}$ and $f \in H_{j l}$ with $(i, k) \neq(j, l)$. In this case, $x \otimes f \in \operatorname{Alg}_{\mathcal{F}} \mathcal{N}$ implies $(i, k)<(j, l)$. So, by step 6 , we have $\Phi(x \otimes f)=0$. Hence we have to prove $\lim _{\lambda} A_{\lambda} x \otimes C_{\lambda} f=0$. For any $\lambda \in \Lambda$ such that $(i, k),(j, l) \in \lambda$, we have

$$
\begin{aligned}
A_{\lambda} x \otimes C_{\lambda} f & =\exp \left(\alpha_{i k} n_{\lambda} t_{\lambda}\right) A_{i k}^{\left(n_{\lambda}\right)} x \otimes \exp \left(-\alpha_{j l} n_{\lambda} t_{\lambda}\right) C_{j l}^{\left(n_{\lambda}\right)} f \\
& =\exp \left(-\left(\alpha_{j l}-\alpha_{i k}\right) n_{\lambda} t_{\lambda}\right) A_{i k}^{\left(n_{\lambda}\right)} x \otimes C_{j l}^{\left(n_{\lambda}\right)} f .
\end{aligned}
$$

Thus $\left\|A_{\lambda} x \otimes C_{\lambda} f\right\| \leq\|x\| \cdot\|f\| \cdot t_{\lambda}^{2} \exp \left(-\left(\alpha_{j l}-\alpha_{i k}\right) n_{\lambda} t_{\lambda}\right)$. Note that $\alpha_{j l}-\alpha_{i k}>0$. So $\lim _{\lambda} t_{\lambda}^{2} \exp \left(-\left(\alpha_{j l}-\alpha_{i k}\right) n_{\lambda} t_{\lambda}\right)=0$, which implies $\lim _{\lambda} A_{\lambda} x \otimes C_{\lambda} f=0$.

Case $3^{\circ} \cdot x=\bigoplus_{(i, k) \in \lambda_{1}} x_{i k}$ and $f=\bigoplus_{(j, l) \in \lambda_{2}} f_{j l}$, for some $\lambda_{1}, \lambda_{2} \in \Lambda$. Since $x \otimes$ $f \in \operatorname{Alg}_{\mathcal{F}} \mathcal{N},(i, k) \leq(j, l)$ holds for all $(i, k) \in \lambda_{1}$ and $(j, l) \in \lambda_{2}$. So $\lambda_{1} \cap \lambda_{2}=\emptyset$ or 
$\lambda_{1} \cap \lambda_{2}=\left\{\left(i_{0}, k_{0}\right)\right\}$, where $\left(i_{0}, k_{0}\right)=\max \left\{(i, k) \mid(i, k) \in \lambda_{1}\right\}$. If $\lambda_{1} \cap \lambda_{2}=\left\{\left(i_{0}\right.\right.$, $\left.\left.k_{0}\right)\right\}$, then

$$
x \otimes f=x_{i_{0} k_{0}} \otimes f_{i_{0} k_{0}}+\sum_{\substack{(i, k) \in \lambda_{1},(j, l) \in \lambda_{2} \\(i, k) \neq(j, l)}} x_{i k} \otimes f_{j l} .
$$

Hence

$$
\begin{aligned}
\lim _{\lambda} A_{\lambda} x \otimes C_{\lambda} f= & \lim _{\lambda} A_{\lambda} x_{i_{0} k_{0}} \otimes C_{\lambda} f_{i_{0} k_{0}} \\
& +\sum_{\substack{(i, k) \in \lambda_{1},(j, l) \in \lambda_{2} \\
(i, k) \neq(j, l)}} \lim _{\lambda} A_{\lambda} x_{i k} \otimes C_{\lambda} f_{j l} .
\end{aligned}
$$

By cases $1^{\circ}$ and $2^{\circ}$, it is easily seen that

$$
\lim _{\lambda} A_{\lambda} x \otimes C_{\lambda} f=\lim _{\lambda} A_{\lambda} x_{i_{0} k_{0}} \otimes C_{\lambda} f_{i_{0} k_{0}}=\Phi\left(x_{i_{0} k_{0}} \otimes f_{i_{0} k_{0}}\right)=\Phi(x \otimes f) .
$$

Similarly, if $\lambda_{1} \cap \lambda_{2}=\emptyset$, one can check that $\lim _{\lambda} A_{\lambda} x \otimes C_{\lambda} f=\Phi(x \otimes f)=0$.

Case $4^{\circ}$. In general, $x \otimes f \in \operatorname{Alg}_{\mathcal{F}} \mathcal{N}$ implies that there exists $\left(i_{0}, k_{0}\right) \in \Omega$ such that $x \in \bigoplus_{(i, k) \leq\left(i_{0}, k_{0}\right)} H_{i k}$ and $f \in \bigoplus_{\left(i_{0}, k_{0}\right) \leq(i, k)} H_{i k}$. Write $x=\bigoplus_{(i, k) \leq\left(i_{0}, k_{0}\right)} x_{i k}$ and $f=\bigoplus_{\left(i_{0}, k_{0}\right) \leq(j, l)} f_{j l}$. Given any positive number $\varepsilon<\|\Phi\|$, there exist $\lambda_{1}$, $\lambda_{2} \in \Lambda$ (we may require $\left(i_{0}, k_{0}\right) \in \lambda_{1} \cap \lambda_{2}$ ) such that $\left\|x-x_{\lambda_{1}}\right\|<\frac{\varepsilon}{3\|\Phi\|(\|x\|+\|f\| \text { ) }}$ and $\left\|f-f_{\lambda_{2}}\right\|<\frac{\varepsilon}{3\|\Phi\|(\|x\|+\|f\|)}$, where $x_{\lambda_{1}}=\bigoplus_{(i, k) \in \lambda_{1}} x_{i k}$ and $f_{\lambda_{2}}=\bigoplus_{(j, l) \in \lambda_{2}} f_{j l}$. So

$$
\left\|\Phi(x \otimes f)-\Phi\left(x_{\lambda_{1}} \otimes f_{\lambda_{2}}\right)\right\|<\|\Phi\|(\|x\|+\|f\|) \frac{\varepsilon}{3\|\Phi\|(\|x\|+\|f\|)}=\frac{\varepsilon}{3} .
$$

By case $3^{\circ}$, there exists $\lambda^{\prime} \supset \lambda_{1} \cup \lambda_{2}$ such that

$$
\left\|A_{\lambda} x_{\lambda_{1}} \otimes C_{\lambda} f_{\lambda_{2}}-\Phi\left(x_{\lambda_{1}} \otimes f_{\lambda_{2}}\right)\right\|<\frac{\varepsilon}{3}
$$

whenever $\lambda \supset \lambda^{\prime}$. For any $\lambda \in \Lambda$ satisfying $\lambda \supset \lambda_{1} \cup \lambda_{2}$, we have

$$
\begin{aligned}
& \left\|A_{\lambda}\left(x_{\lambda_{1}}-x\right) \otimes C_{\lambda} f_{\lambda_{2}}\right\| \\
& =\left\|\sum_{(i, k) \in \lambda \backslash \lambda_{1}(j, l) \in \lambda_{2}} A_{\lambda} x_{i k} \otimes C_{\lambda} f_{j l}\right\| \\
& =\left\|\sum_{(i, k) \in \lambda \backslash \lambda_{1}(j, l) \in \lambda_{2}} \sum_{\sum} \exp \left(-\left(\alpha_{j l}-\alpha_{i k}\right) n_{\lambda} t_{\lambda}\right) A_{i k}^{\left(n_{\lambda}\right)} x_{i k} \otimes C_{j l}^{\left(n_{\lambda}\right)} f_{j l}\right\| \\
& \leq \sum_{(i, k) \in \lambda \backslash \lambda_{1}(j, l) \in \lambda_{2}} \exp \left(-\left(\alpha_{j l}-\alpha_{i k}\right) n_{\lambda} t_{\lambda}\right) \cdot t_{\lambda}^{2}\left\|x_{i k}\right\| \cdot\left\|f_{j l}\right\| \\
& <\frac{\varepsilon}{3\|\Phi\|(\|x\|+\|f\|)}\|f\| t_{\lambda}^{2} \exp \left(-\delta n_{\lambda} t_{\lambda}\right) \cdot \sum_{(i, k) \in \lambda \backslash \lambda_{1}} \sum_{(j, l) \in \lambda_{2}} 1 \\
& \leq \frac{\varepsilon}{3\|\Phi\|(\|x\|+\|f\|)}\|f\| t_{\lambda}^{2} n_{\lambda}^{2} \exp \left(-\delta n_{\lambda} t_{\lambda}\right),
\end{aligned}
$$

where $0<\delta=\alpha_{i_{0} k_{0}}-\alpha_{i_{0}\left(k_{0}-1\right)} \leq \min \left\{\left(\alpha_{j l}-\alpha_{i k}\right) \mid(i, k) \in \lambda \backslash \lambda_{1}\right.$ and $\left.(j, l) \in \lambda_{2}\right\}$. Since $\lim _{\lambda} t_{\lambda}^{2} n_{\lambda}^{2} \exp \left(-\delta n_{\lambda} t_{\lambda}\right)=0$, there exists $\lambda^{\prime \prime} \supset \lambda_{1} \cup \lambda_{2}$ such that $t_{\lambda}^{2} n_{\lambda}^{2} \exp \left(-\delta n_{\lambda} t_{\lambda}\right)<\varepsilon$ whenever $\lambda>\lambda^{\prime \prime}$. Therefore

$$
\left\|A_{\lambda}\left(x_{\lambda_{1}}-x\right) \otimes C_{\lambda} f_{\lambda_{2}}\right\|<\frac{\|f\| \varepsilon^{2}}{3\|\Phi\|(\|x\|+\|f\|)}
$$


whenever $\lambda>\lambda^{\prime \prime}$. For $\lambda \supset \lambda_{1} \cup \lambda_{2}$, we have

$$
\begin{aligned}
& \left\|A_{\lambda} x \otimes C_{\lambda}\left(f_{\lambda_{2}}-f\right)\right\| \\
= & \left\|-\sum_{(i, k) \in \lambda(j, l) \in \lambda \backslash \lambda_{2}} \sum_{\lambda} x_{i k} \otimes C_{\lambda} f_{j l}\right\| \\
= & \left\|-\sum_{(i, k) \in \lambda(j, l) \in \lambda \backslash \lambda_{2}} \exp \left(-\left(\alpha_{j l}-\alpha_{i k}\right) n_{\lambda} t_{\lambda}\right) A_{i k}^{\left(n_{\lambda}\right)} x_{i k} \otimes C_{j l}^{\left(n_{\lambda}\right)} f_{j l}\right\| \\
< & \sum_{(i, k) \in \lambda(j, l) \in \lambda \backslash \lambda_{2}} \exp \left(-\left(\alpha_{j l}-\alpha_{i k}\right) n_{\lambda} t_{\lambda}\right) \cdot t_{\lambda}^{2}\|x\| \frac{\varepsilon}{3\|\Phi\|(\|x\|+\|f\|)} \\
\leq & \frac{\varepsilon}{3\|\Phi\|(\|x\|+\|f\|)}\|x\| t_{\lambda}^{2} n_{\lambda}^{2} \exp \left(-\delta_{1} n_{\lambda} t_{\lambda}\right),
\end{aligned}
$$

where $0<\delta_{1}=\alpha_{i_{0}\left(k_{0}+1\right)}-\alpha_{i_{0} k_{0}}=\min \left\{\left(\alpha_{j l}-\alpha_{i k}\right) \mid(i, k) \in \lambda\right.$ and $(j, l) \in$ $\left.\lambda \backslash \lambda_{2}\right\}$. Since $\lim _{\lambda} t_{\lambda}^{2} n_{\lambda}^{2} \exp \left(-\delta_{1} n_{\lambda} t_{\lambda}\right)=0$, there exists $\lambda^{\prime \prime \prime} \supset \lambda_{1} \cup \lambda_{2}$ such that $t_{\lambda}^{2} n_{\lambda}^{2} \exp \left(-\delta_{1} n_{\lambda} t_{\lambda}\right)<\varepsilon$ whenever $\lambda>\lambda^{\prime \prime \prime}$. Therefore $\left\|A_{\lambda} x \otimes C_{\lambda}\left(f_{\lambda_{2}}-f\right)\right\|<$ $\frac{\|x\| \varepsilon^{2}}{3\|\Phi\|(\|x\|+\|f\|)}$ whenever $\lambda>\lambda^{\prime \prime \prime}$. It follows that, for any $\lambda$ with $\lambda>\lambda^{\prime \prime} \cup \lambda^{\prime \prime \prime}$, we have

$$
\begin{aligned}
& \left\|A_{\lambda} x_{\lambda_{1}} \otimes C_{\lambda} f_{\lambda_{2}}-A_{\lambda} x \otimes C_{\lambda} f\right\| \\
\leq & \left\|A_{\lambda}\left(x_{\lambda_{1}}-x\right) \otimes C_{\lambda} f_{\lambda_{2}}\right\|+\left\|A_{\lambda} x \otimes C_{\lambda}\left(f_{\lambda_{2}}-f\right)\right\| \\
\leq & \frac{\varepsilon}{3\|\Phi\|(\|x\|+\|f\|)}\|x\| \varepsilon+\frac{\varepsilon}{3\|\Phi\|(\|x\|+\|f\|)}\|f\| \varepsilon \\
< & \frac{\varepsilon}{3} .
\end{aligned}
$$

Let $\lambda_{0}=\lambda^{\prime} \cup \lambda^{\prime \prime} \cup \lambda^{\prime \prime \prime}$. Then for any $\lambda$ with $\lambda>\lambda_{0}$, one has

$$
\begin{aligned}
\| \Phi(x & \otimes f)-A_{\lambda} x \otimes C_{\lambda} f \| \\
\leq & \left\|\Phi(x \otimes f)-\Phi\left(x_{\lambda_{1}} \otimes f_{\lambda_{2}}\right)\right\|+\left\|A_{\lambda} x_{\lambda_{1}} \otimes C_{\lambda} f_{\lambda_{2}}-\Phi\left(x_{\lambda_{1}} \otimes f_{\lambda_{2}}\right)\right\| \\
& +\left\|A_{\lambda} x_{\lambda_{1}} \otimes C_{\lambda} f_{\lambda_{2}}-A_{\lambda} x \otimes C_{\lambda} f\right\| \\
& <\varepsilon .
\end{aligned}
$$

Thus $\Phi(x \otimes f)=\lim _{\lambda} A_{\lambda} x \otimes C_{\lambda} f$ holds for every $x \otimes f \in \operatorname{Alg}_{\mathcal{F}} \mathcal{N}$. Let $B_{\lambda}=C_{\lambda}^{*}$. Then $\Phi(x \otimes f)=\lim _{\lambda} A_{\lambda}(x \otimes f) B_{\lambda}$ holds for every $x \otimes f \in \operatorname{Alg}_{\mathcal{F}} \mathcal{N}$. Since every finite rank operator in $\operatorname{Alg}_{\mathcal{F}} \mathcal{N}$ can be represented as a linear combination of rank one operators in $\operatorname{Alg}_{\mathcal{F}} \mathcal{N}$ and $\Phi$ is linear, $\Phi(F)=\lim _{\lambda} A_{\lambda} F B_{\lambda}$ holds for all $F \in \operatorname{Alg}_{\mathcal{F}} \mathcal{N}$.

Now it is clear that the first half of (2) is true by [7] Theorem 10], which states that a linear map $\varphi$ from a subspace $\mathcal{R}$ of $\mathcal{B}(H)$ into $\mathcal{B}(K)$ is a strong limit of a net of elementary operators of length 1 if and only if $\left.\varphi\right|_{\mathcal{R} \cap \mathcal{F}(H)}$ has the same property. The second half of (2) is easily checked.

We remark that, from the proof of the main theorem, $\Phi$ is completely rank nonincreasing if and only if $\Phi_{2}$ is rank-1 nonincreasing. It is easy to see that these conditions are also equivalent to one that $\Phi_{k+1}$ is rank- $k$ nonincreasing for some positive integer $k$. It is also easy to get some characterizations of the bounded and completely rank preserving linear maps from $\operatorname{Alg} \mathcal{N}$ into $\mathcal{B}(K)$ by applying the main theorem.

Recall that a linear map $\Phi: \mathcal{A} \subseteq \mathcal{B}(H) \rightarrow \mathcal{B}(K)$ is said to be completely $k$-rank nonincreasing if, for each positive integer $n, \Phi_{n}$ is $k$-rank nonincreasing. Motivated by the main theorem and the results concerning the characterization of elementary operators in [4] and [5], the following conjecture seems reasonable.

Conjecture. Let $\Phi: \operatorname{Alg} \mathcal{N} \rightarrow \mathcal{B}(K)$ be a bounded linear map. Then $\Phi$ is completely $k$-rank nonincreasing if and only if it is a strong limit of a net of elementary operators of length not greater than $k$. 


\section{ACKNOWLEDGEMENT}

The authors wish to give their thanks to Professor Don Hadwin for his worthwhile suggestions on this paper.

\section{REFERENCES}

1. Chooi, W. and Lim, M., Linear preservers on triangular matrices, Linear Algebra Appl., 269 (1998), 241-255. MR 98m:15007

2. Cui, J., Hou, J., and Li, B., Linear preservers on upper triangular operator matrix algebras, Linear Algebra Appl., 336 (2001), 29-50. MR 2002g:47074

3. Davidson, K. R., Nest Algebras, Pitman Research Notes in Mathematics, Vol. 191, Longman Scientific and Technical, London/New York, 1988. MR 90f:47062

4. Ge, L., Hadwin, D., Hou, J., and Li, J., Rank nonincreasing linear maps on operator spaces, preprint.

5. Hadwin, D. and Larson, D., Completely rank nonincreasing linear maps, J. Funct. Anal., 199 (2003), 210-227.

6. Hadwin, D. and Larson, D., Strong limits of similarities, Operator Theory: Advances and Applications, 104, Birkhäuser-Verlag, Basel, Switzerland, 1998, pp. 139-146. MR 99e:47040

7. Hou, J., Rank-preserving linear maps on $\mathcal{B}(X)$, Sci. China Ser. A 32 (1989), 929-940. MR 92b:47052

8. Hou, J. and Cui, J., Rank-1 preserving linear maps on nest algebras, Linear Algebra Appl., 369 (2003), 263-277.

9. Molnar, L. and Semrl, P., Some linear preserver problems on upper triangular matrices, Linear and Multilinear Algebra, 45 (1998), 189-206. MR 99h:15003

10. Wei, S. and Hou, S., Rank preserving maps on nest algebras, J. Operator Theory, 39 (1998), 207-217. MR 2000m:47099

Department of Mathematics, Shanxi Teachers University, Linfen, 041004, People's Republic of China

Current address: Department of Mathematics, Shanxi University, Taiyuan 030000, People's Republic of China

E-mail address: jhou@dns.sxtu.edu.cn

School of Mathematical Sciences, Peking University, Beijing 100871, People's RePUBLIC OF CHINA

Current address: Department of Mathematics, Shanxi Teachers University, Linfen, 041004, People's Republic of China

E-mail address: cuijl@dns.sxtu.edu.cn 